\title{
L'ambition métropolitaine : clé vers un aménagement du territoire transfrontalier en Grande Région SaarLorLux ?
}

\author{
Par Estelle Evrard et Christian Schulz
}

Estelle Evrard, université du Luxembourg, unité de recherche IPSE, Institut de géographie et aménagement du territoire - estelle.evrard@uni.lu

Christian Schulz, université du Luxembourg, unité de recherche IPSE, Institut de géographie et aménagement du territoire-Christian.schulz@uni.lu

\section{Introduction}

Sous l'impulsion notamment d'une intégration européenne de plus en plus importante, le phénomène de métropolisation concerne désormais une dizaine d'espaces transfrontaliers (ESPON, Metroborder, 2010). Le travail frontalier, qui y participe, ne constitue qu'une caractéristique des liens fonctionnels de plus en plus prégnants liant nombre d'espaces frontaliers. Mobilité et accessibilité, attractivité économique, étalement urbain, mobilité résidentielle sont autant de phénomènes liés générant d'importants impacts interrégionaux sur le développement territorial. Leur ampleur est telle qu'une concertation et une coordination politique transfrontalière accrues s'avèrent essentielles afin de faire face aux dynamiques spatiales qu'ils engendrent et d'anticiper leurs impacts nuisibles. En l'absence d'une compétence en la matière (Waterhout, 2008), l'échelle européenne a soutenu essentiellement financièrement la coopération transfrontalière et interrégionale par le biais de ses fonds structurels (ex. programmes opérationnels INTERREG, Blatter 2004 ; Perkmann 2003 ; 2007a). Depuis 2008, à ce soutien financier est venu s'ajouter l'outil juridique du GECT (Groupement européen de coopération territoriale) permettant la mise sur pied d'un organisme commun transfrontalier, capable de mener des projets sur l'ensemble de l'espace de coopération des partenaires associés (EC, 2008).

Pourtant, le domaine de l'aménagement du territoire dans le contexte transfrontalier demeure complexe. Déjà conflictuel dans un contexte national ou infranational et marqué par des intérêts de pouvoir et des rivalités économiques, cette coopération est d'autant plus difficile que les structures administratives et les instruments de planification peuvent se révéler incompatibles (Schulz 
2013). Au niveau européen, l'application du principe de subsidiarité ne vient pas atténuer les différences entre les systèmes de planification nationale.

La présente contribution se concentre sur le cas de la Grande Région SaarLorLux particulièrement emblématique puisqu'elle représente l'espace transfrontalier le plus vaste d'Europe et celui concentrant le plus grand nombre de travailleurs frontaliers en Europe. Elle se caractérise également par sa complexité institutionnelle : cinq entités géographiques - Lorraine, Luxembourg, Rhénanie-Palatinat, Sarre, Wallonie - issues de quatre pays déploient leurs compétences sur trois niveaux administratifs différents, local, régional et national. La complexité mais aussi le caractère stratégique de la coopération dans le domaine de l'aménagement du territoire seront illustrés par la stratégie Metroborder, la plus récente initiative adoptée dans ce domaine, visant à établir une véritable stratégie territoriale comprenant une approche polycentrique et métropolitaine. Le projet de développer un « schéma de développement territorial » commun illustre l'ambition politique sous-tendant cette stratégie ainsi que l'ampleur de la tâche. Cette démarche peut être comprise comme une initiative volontariste et ambitieuse. Elle vise à tenir compte tant des réalités internes comme par exemple l'ampleur des flux transfrontaliers et la reconnaissance tacite de l'existence d'un espace central en Grande Région SaarLorLux, qu'externe comme en particulier de sa situation géographique, spatialement entourée de métropoles plus compétitives.

\section{- Les multiples enjeux de l'aménagement du territoire dans le contexte transfrontalier}

Dans un contexte purement national, l'aménagement du territoire constitue un champ politique transversal, potentiellement conflictuel puisqu'il doit intégrer des intérêts très divergents. Ce défi est exacerbé dans le contexte transfrontalier puisque le nombre d'acteurs associés et d'intérêts impliqués est démultiplié par le nombre d'entités associées. L'absence d'une politique commune et d'une compétence supranationale européenne ne favorise pas non plus la coordination des différentes approches nationales en la matière (Dühr, Colomb, Nadin 2010 ; Faludi 2008). Après l'échec du Schéma de Développement de l'Espace Communautaire (SDEC) à la fin des années 1990, les développements autour de la cohésion territoriale n'ont pas (encore) su établir un cadre d'orientation traitant de la dimension transfrontalière de l'aménagement. Ce qui vaut globalement pour l'échelle interétatique européenne se reflète également au niveau interrégional où les cadres de référence politiques et législatifs restent principalement limités au contexte national.

Malgré le grand nombre d'études empiriques sur les phénomènes spatiaux et la gouvernance transfrontalière, les travaux traitant explicitement du champ 
de l'aménagement sont moins fréquents. Parmi les exceptions, on trouve Perkmann (2007b), Renard (2010; et le numéro spécial de la revue Mosella qui suit son avant-propos), Knippschild (2011), Hinfray (2010) ou encore Ritter (2010). L'aménagement du territoire étant une politique transversale impliquant plusieurs niveaux administratifs et plusieurs types d'acteurs (publics, économiques, associatifs/ONG), cette politique correspond largement au « Type 2 » de gouvernance multi-échelle proposée par Hooghe/Marks (2003). Dans le contexte transfrontalier, le nombre de partenaires, de niveaux impliqués et de responsabilités souvent asymétriques entre les homologues gouvernementaux et administratifs ne fait que complexifier encore la coopération horizontale (Chilla/Evrard/Schulz 2012).

Le développement d'une approche commune en termes d'aménagement du territoire dans un espace frontalier est ainsi une entreprise particulièrement complexe pour diverses raisons :

- Cadres juridiques asymétriques : la place formelle de l'aménagement du territoire, le mandat des institutions ainsi que les instruments mis à leur disposition varient d'une région à l'autre. Il s'agit bien plus que d'un problème technique (par exemple, synchronisation de procédures administratives afin de pouvoir coordonner la révision des plans régionaux respectifs); il comprend la difficulté, voire l'incapacité actuelle de nombreux acteurs de transposer des schémas de développement interrégionaux en plans formels et contraignants dans leur région (Spannowsky, 2005-2007; Comte, Levrat, 2006). Cette difficulté est d'autant plus importante que les partenaires institutionnels ne peuvent fonder leur action sur un cadre institutionnel transfrontalier ferme, voire une autorité supra-régionale capable d'assurer la mise en œuvre des décisions communes (Evrard à paraître). Les organismes de coopération interrégionale en Grande Région SaarLorLux constituent des plates-formes d'échange et de concertation (parfois de décision). Elles n'ont pas la possibilité de mettre en œuvre les décisions ou de s'assurer qu'elles sont suivies d'effet chez les entités partenaires.

- Différentes cultures de planification : les obstacles de nature formelle comme les décalages législatifs sont aggravés par les différences considérables entre les «philosophies »d'aménagement du territoire. Ces dernières imprègnent le contexte de chaque entité, et peuvent conduire à des incompatibilités d'approches, même s'il y a consensus sur la démarche stratégique (Ritter, 2010 : 118 ; Othengrafen, Reimer, 2013 ; Chilla, Schulz, 2014).

Il résulte de ce contexte une grande complexité, en particulier dès lors qu'une frontière administrative importante découpe le territoire. Ce phénomène que l'on peut observer à l'intérieur d'un pays (Hoyler, Freytag, Mager, 2006) vaut d'autant plus pour des frontières étatiques. Étant donné son contexte multilatéral (4 pays), la situation en Grande Région SaarLorLux 
est particulière en ce qui concerne le nombre de partenaires, les langues, les cultures et objectifs politiques (fig. 1).

\section{- Dépasser l'asymétrie institutionnelle et mettre en place une politique coordonnée d'aménagement du territoire en Grande Région SaarLorLux}

La structure institutionnelle des partenaires associés à la Grande Région SaarLorLux est particulièrement hétérogène, ce qui complexifie la mise en place d'une politique coordonnée d'aménagement du territoire.

\section{Une importante asymétrie institutionnelle}

La Grande Région SaarLorLux réunit à elle seule quatre états dont deux sont fédéraux et deux sont centralisés, avec la spécificité des communautés linguistiques en Wallonie et d'une décentralisation toujours plus renforcée en France. Malgré son caractère simplificateur, la figure 1 illustre cette asymétrie institutionnelle, en représentant la variété d'acteurs et d'échelles formellement chargés de la planification spatiale en Grande Région SaarLorLux. Le niveau national joue un rôle central en France où le Préfet de Région représente l'État en la matière ainsi qu'au Luxembourg où le département de l'aménagement du territoire au niveau ministériel est en charge de l'ensemble du territoire national (le niveau administratif régional est absent étant donné la taille du pays). Dans les systèmes fédéraux allemand et belge, les responsabilités (législatives et exécutives) sont déléguées aux Länder de Rhénanie-Palatinat et de Sarre ainsi qu'à la Wallonie. La Communauté germanophone de Belgique se verra vraisemblablement attribuer des compétences dans ce domaine dans un avenir proche.

Le niveau régional, c'est-à-dire le niveau administratif intermédiaire entre les municipalités et l'État, est particulièrement hétérogène. Soit il est faiblement institutionnalisé comme en France où le pouvoir des départements et des régions dans ce domaine dépend fortement de l'État central, soit il n'est pas impliqué formellement comme dans le cas des provinces en Belgique, ou encore il est simplement absent, du fait de l'inexistence du niveau administratif correspondant comme au Luxembourg et en Sarre. Ce niveau se révèle fortement impliqué uniquement en Rhénanie-Palatinat où les Planungsregionen exercent leurs compétences respectives à ce niveau intermédiaire.

Malgré d'importantes différences en termes de nombre et de taille des communes, c'est au niveau local que l'on compte les plus grandes similarités législatives et opérationnelles. Pourtant, ce niveau est moins important pour la coordination interrégionale. 
Fig. 1 : Niveaux administratifs potentiellement impliqués au sein de la Grande Région SaarLorLux (Schulz 2013: 117)

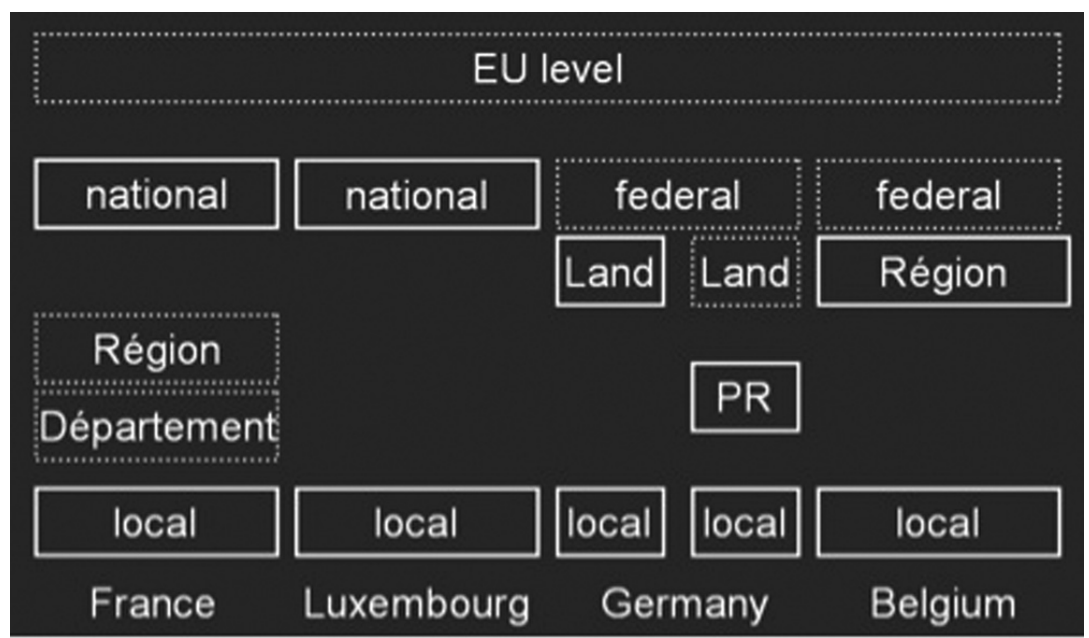

Cases en gras : compétences en matière d'aménagement du territoire ; PR = Planungsregion en Rhénanie-Palatinat.

\section{Quatre décennies en quête d'une politique coordonnée d'aménagement du territoire}

Malgré une coopération transfrontalière longue de plus de 45 ans en Grande Région SaarLorLux (Clément, 2015), les achèvements tangibles dans le domaine de l'aménagement du territoire restent peu nombreux. Ils se limitent surtout à des projets d'infrastructure locaux (tracé de la Saarbahn SaarbrückenSarreguemines, ponts et passerelles, réseaux de pistes cyclables). Plus récemment, quelques conventions bilatérales et sectorielles ont été établies, comme par exemple le schéma stratégique de mobilité transfrontalière LuxembourgLorraine (SMOT), comprenant de nouvelles orientations à court, moyen et long terme (ministère des Transports du Grand-Duché de Luxembourg, conseil régional de Lorraine, CFL, SNCF 2008).

Pourtant, nombreuses ont été les initiatives plus cohérentes ; la plus ambitieuse d'entre elles étant vraisemblablement le « Schéma de développement de l'Espace SaarLorLux + ». Lancé à la fin des années 1990 à l'initiative du Luxembourg et de la Sarre, ce schéma résultait d'une étude intégrée pour l'ensemble des partenaires institutionnels. Il représente le premier essai sérieux visant à 1) traduire des objectifs européens en un schéma d'aménagement (Leitbild) régional, et à 2) développer une stratégie de moyen terme (sur 20 ans) basée sur des analyses de tendances spatiales et sur des scénarios. «Pour diverses raisons » (Vidal, Niedermeyer 2011: 303), entre autres par manque de reconnaissance de certains partenaires, l'étude n'a pas été publiée officiellement et, ainsi, n'a pas eu d'impact sur les débats politiques. Un autre document clé lancé en 2002 
par les mêmes partenaires (Sarre et Luxembourg) sous forme de la «Vision d'Avenir $2020 »$ avait un focus thématique beaucoup plus large. Il formulait des orientations générales et des objectifs de long terme, délibérément sans spécifier la dimension géographique des actions proposées. Même si elle bénéficiait d'un plus grand soutien politique, la « Vision d'Avenir $2020 »$ n'a pas su compenser le manque d'une stratégie commune en matière d'aménagement du territoire.

De la double absence d'une stratégie politique commune et d'un cadre réglementaire permettant de répondre aux défis actuels en termes de développement spatial découle un vide institutionnel (institutional void, Hajer 2003 : 175). Celui-ci s'articule au moins autour de trois problématiques :

- Le manque combiné d'un mandat politique, de moyens budgétaires et/ou de base juridique expliquent le fait qu'une grande partie des problèmes pertinents ne soient pas pris en main de manière appropriée. L'urbanisation transfrontalière peu coordonnée, liée à la mobilité résidentielle, ainsi que les transports publics peu intégrés en constituent des exemples criants.

- Le manque de concertation et de coordination se traduit également par une allocation inefficace de ressources sous forme de double, voire de multiples emplois. On peut observer une sous-utilisation de certaines infrastructures publiques, où des investissements partagés par les partenaires pourraient mener à des résultats plus bénéfiques pour l'ensemble des parties. Par exemple, l'armature aéroportuaire de la Grande Région SaarLorLux ne comprend pas moins de sept aéroports dits internationaux (CharleroiBruxelles-Sud (BE), Liège (BE), Luxembourg-Findel (LU), Metz-NancyLorraine (FR), Saarbrücken-Ensheim (DE), Frankfurt-Hahn (DE), Zweibrücken $^{1}(\mathrm{DE})$ ) ; la plupart étant fortement subventionnée par les autorités nationales ou régionales.

- Comme le montre l'évolution du secteur des commerces de détail visant une clientèle outre-frontière, certains investisseurs et entreprises profitent délibérément des ambiguïtés, voire des contradictions réglementaires. Ces pratiques aggravent les rivalités économiques et politiques entre les partenaires. La carte (fig. 2) montre le nombre de « grandes surfaces » et centres d'achat $\left(>7000 \mathrm{~m}^{2}\right)$ établis ces dernières années le long de la frontière luxembourgeoise afin de capter le pouvoir d'achat des frontaliers ou des résidents luxembourgeois faisant fréquemment leurs courses à l'extérieur du pays pour profiter des différentiels de prix (Wille 2015).

1. Le $1^{\text {er o }}$ ctobre 2014, la Commission européenne a décidé que les subsides versés par le Land de RhénaniePalatinat à la société exploitant l'aéroport de Zweibrücken sont des aides d'état non justifiées selon le droit communautaire (Commission 2014). L'aéroport fermera vraisemblablement ses portes sous peu car il semble incapable de rembourser les 47 millions d'Euros d'aides indûment perçues. Le même jour, une autre décision concernait l'aéroport de Charleroi pour lequel une partie seulement de l'aide accordée a été considérée comme légale, un montant de 6 millions d'Euros devra être remboursé 
Fig. 2 : Grandes surfaces et centres d'achat $\left(>7000 \mathrm{~m}^{2}\right)$ à proximité des frontières du Grand-Duché de Luxembourg (Affolderbach 2013: 134)

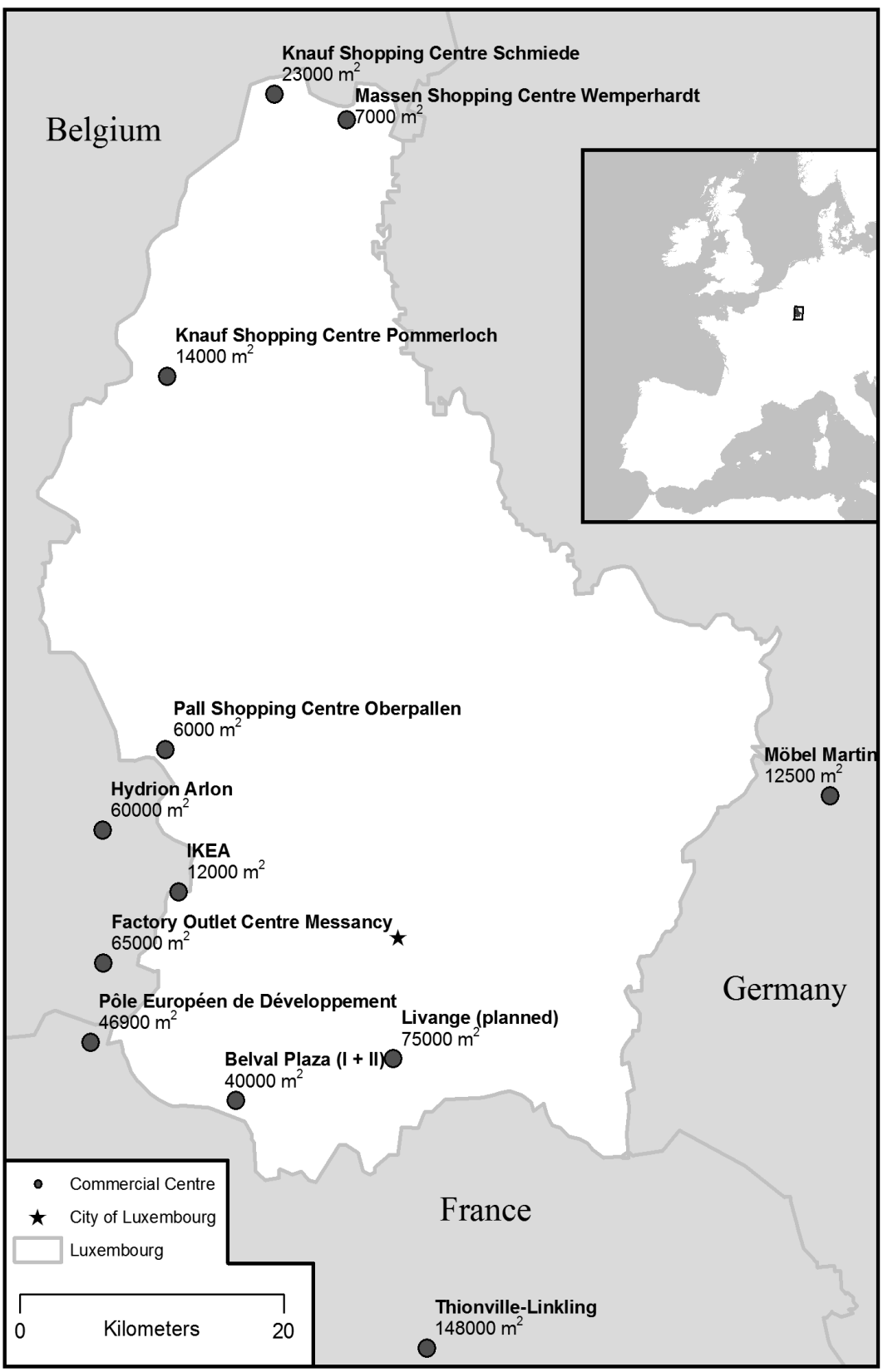


Ces dernières années, les problèmes de transports routiers, de mobilité résidentielle et d'étalement urbain liés à des effets frontières croissants ont considérablement augmenté, en particulier au Luxembourg et dans ses espaces limitrophes. Ils sont d'ailleurs régulièrement dénoncés dans les médias ainsi que par des associations d'utilisateurs (travailleurs frontaliers). Aussi, il est peu surprenant que le Grand-Duché ait pris l'initiative de relancer les efforts visant à une meilleure coordination en termes d'aménagement du territoire. En faisant de ce domaine la première priorité de sa présidence du Sommet de la Grande Région (2008-2009), il est notamment à l'origine de l'initiative de créer une Région métropolitaine polycentrique transfrontalière (RMPT). Cette dernière est également connue sous le libellé Metroborder, dénommant à la fois un projet scientifique dans le cadre du programme ESPON ainsi qu'une stratégie politique entamée par les partenaires du Sommet. Elle sera présentée plus en détail dans la partie suivante.

\section{Le CAS de l'OUVERTURe d'une SUCCURSALe d'IKEA À SterPenich/ArLon (BE), 2005}

En 2005, IKEA a ouvert une succursale à Sterpenich/Arlon, au bord de l'autoroute LuxembourgBruxelles sur un terrain vierge touchant littéralement le territoire du Luxembourg et son paysage agricole. Initialement, l'entreprise envisageait de s'installer au Grand-Duché. Cette installation a été empêchée par le « moratoire grandes-surfaces » en vigueur au Luxembourg entre 1997 et 2006, prohibant l'ouverture de tout commerce ayant une surface de vente de plus de $10000 \mathrm{~m}^{2}$ sur des sites extra-urbains. Près de deux tiers des clients d'IKEA Sterpenich proviennent du Luxembourg, un quart seulement de Belgique (Affolderbach 2013: 132).

La polémique autour de cette localisation était assez vive au Grand-Duché (« Il faut éviter un deuxième IKEA ! »). Les autorités nationales ont décidé d'abroger le moratoire en 2006. Était lancée simultanément une stratégie globale visant à faire du Luxembourg « le centre d'achat de la Grande-Région » (Juncker 2006). Ironiquement, en 2014, le conseil municipal d'Arlon votait contre l'extension prévue de la zone commerciale créée pour IKEA. Du côté luxembourgeois, six communes voisines - réunies dans un syndicat intercommunal - se mettaient d'accord sur le développement de la « zone d'activités économiques à caractère régional dans la région de l'ouest du pays » (ZARO) à Grass/Steinfort (20 ha de surfaces nettes) - à quelques mètres seulement du site IKEA et sur un terrain jusqu'à présent agricole et non intégré dans un espace urbanisé.

\section{- Vers une « Région métropolitaine polycentrique transfrontalière ॥ ? Entre ambitions et réalisations}

En 2008, le Sommet des Exécutifs, principale instance de coopération politique et institutionnelle au niveau régional, a impulsé la stratégie Metroborder. L'objectif sur le long terme est la « mise en place progressive d'une métropole polycentrique, transfrontalière, capable de se mesurer avec les grandes métropoles nationales » (Sommet 2008: 9). À cet objectif fort ambitieux est accolé un objectif à court terme, qui l'est tout autant : «dégager [...] une démarche 


\section{Photo 1 : Vue aérienne de la succursale IKEA à Sterpenich/Arlon, à proximité immédiate de la frontière luxembourgeoise (Schulz, Chilla)}

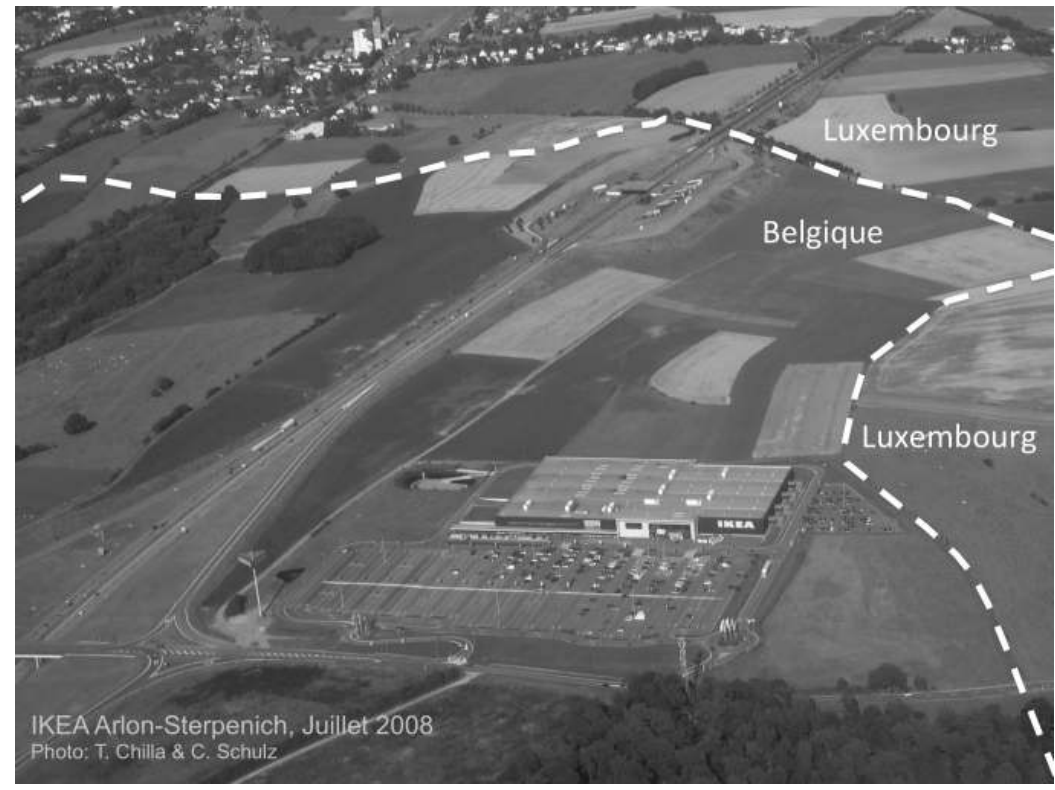

cohérente et intégrative en matière de développement territorial et de planification territoriale tant de l'espace urbain que de l'espace rural » (ibid.). En 2015, après sept ans et quatre présidences du Sommet des Exécutifs, instance politique la plus significative dans cet espace, force est de constater que quelques importants jalons ont été posés. Ils alimentent essentiellement l'objectif que les partenaires institutionnels s'étaient fixé sur le court terme. Le premier est institutionnel. Un Groupement Européen de Coopération Territoriale (GECT) a été créé et mis en place, il vise à faciliter l'organisation et le suivi des Sommets. Son caractère transversal aux groupes de travail du Sommet a pour but de faciliter les synergies entre eux tout en évitant les incohérences ou doublons. Le second est d'ordre instrumental. La mise en place d'un Système d'information géographique (SIG) commun, nommé SIG-GR, va au-delà de la mise en réseau des instituts régionaux de statistiques pour permettre la mise en place d'un système intégré d'appréhension de l'espace transfrontalier. Ces travaux constituent des avancées incontestables dans la mesure où ils contribuent à institutionnaliser une échelle transfrontalière se distinguant de l'addition des partenaires institutionnels. Ils constituent une pierre importante à l'édifice que constitue la construction politique de la Grande Région. Toutefois, l'objectif concret de concevoir ce qui est maintenant nommé « schéma de développement territorial de la Grande Région » reste en discussion. Afin de prendre toute la 
mesure de ces différentes avancées et d'analyser les ressorts de ce positionnement politique transfrontalier, nous reviendrons dans un premier temps sur les enjeux de la stratégie Metroborder.

\section{Devenir métropole transfrontalière : ambition européenne et recherche d'un projet transfrontalier fédérateur}

«Faisant le constat de l'absence dans la Grande Région d'une véritable métropole au sens européen du terme, le Sommet appuie résolument le projet considéré comme primordial pour l'avenir de l'espace de coopération d'autant plus que la Commission européenne souhaite, conformément à la stratégie de Lisbonne, renforcer les métropoles existantes et soutenir les projets de création de métropoles ou de régions métropolitaines. Ainsi, le projet permettra de dégager une stratégie conjointe permettant à la Grande Région :

- de mieux rivaliser avec les grandes aires métropolitaines européennes ;

- d'augmenter son potentiel et son attrait économique ;

- de promouvoir un développement spatial polycentrique tout en créant de nouvelles relations ville-campagne ;

- d'assurer un accès équivalent au savoir et aux infrastructures tout en renforçant les réseaux de formation et de recherche. » (Sommet 2009: 7)

Cet extrait de la déclaration du Sommet révèle clairement que le positionnement politique sous-tendant la stratégie Metroborder a plusieurs vocations pouvant s'avérer difficilement conciliables dans leur concrétisation. D'une part, elle se revendique essentiellement métropolitaine pour mieux se positionner au niveau européen. D'autre part, elle se veut inclusive territorialement en étant polycentrique et thématiquement large en touchant à la fois les infrastructures, la recherche et l'économie. Dans cette construction «métropolitaine, polycentrique et transfrontalière », le qualificatif «transfrontalière » est un pléonasme puisqu'il vise essentiellement à rappeler à l'extérieur la singularité de cet espace. Nous nous concentrons donc dans un premier temps sur la portée de l'ambition métropolitaine qui renvoie aux choix politiques et à la direction stratégique que la Grande Région SaarLorLux se propose de suivre. Dans un second temps, nous analyserons les enjeux de l'adjectif «polycentrique », qui vise essentiellement à garantir à chaque partenaire le caractère spatialement inclusif de ce processus. Au total, cette stratégie Metroborder, nouvelle construction politique dans le paysage grand-régional, laisse entrevoir plusieurs visions pour l'avenir de la coopération dans cet espace.

\section{Une « fièvre métropolitaine » contagieuse ?}

La métaphore de Leber et Kunzmann (2006), utilisée pour décrire le contexte allemand, peut être utilisée également pour caractériser le contexte européen et les pays associés à la coopération transfrontalière en Grande Région SaarLorLux. De fait, dès 1999, au niveau européen, le Schéma de Développement de 
l'Espace Communautaire (SDEC) met en avant le rôle charnière des villes dans un système d'interdépendances multi-niveaux, facilitant leur intégration dans les réseaux européens et mondiaux (SDEC 1999 : 7). En étant « facteur d'intégration fonctionnelle transfrontalière » (ibid. : 22), les villes sont considérées comme essentielles dans la concrétisation du marché unique. Dans ce contexte, les autorités infranationales (indistinctement régions ou villes) deviennent une échelle incontournable de la politique régionale communautaire (ibid. : 48). Les autorités infra-étatiques sont quant à elles incitées à renforcer leur coopération et à développer un positionnement «stratégique » propre auprès de l'Union Européenne (Sykes 2008 : 55). Cette approche a été confirmée récemment par l'Agenda territorial 2020 qui met en exergue leur fonction de nœud, garantissant d'une part un positionnement dans les flux mondiaux et d'autre part participant à l'économie locale (Agenda territorial 2020 2011: 5). La stratégie Metroborder intervient dans ce contexte comme une initiative visant à coordonner le développement territorial aux marges de plusieurs États.

Ce discours politique s'est également manifesté dans les quatre pays associés à la coopération transfrontalière de la Grande Région SaarLorLux.

- En France, celui-ci s'est en particulier manifesté par la création de deux nouvelles structures administratives en 2010, la métropole et le pôle métropolitain, qui peuvent recevoir des collectivités territoriales les ayant initiées à des compétences à vocation métropolitaine (développement économique, innovation, recherche, enseignement supérieur). Ces structures peuvent concentrer en une entité des compétences propres à stimuler la compétitivité d'un espace (CGCT, Art. L.5731-1 al.1). Le Sillon lorrain (agglomérations de Thionville, Metz, Nancy et Épinal) a ainsi été institutionnalisé sous la forme d'un pôle métropolitain en 2011. Il se positionne clairement comme structurant en son sein le potentiel métropolitain du côté français de la Grande Région SaarLorLux et exerce à ce titre une influence dans les débats relatifs à la stratégie Metroborder.

- En Allemagne, cette « fièvre métropolitaine» (Leber, Kunzman 2006 : 58) date du milieu des années 1990. Six villes allemandes ont d'abord été qualifiées de Métropole d'envergure européenne (Europäische Metropolregion) par la conférence des ministres de l'aménagement du territoire en 1995 (BmBau 1995 : 28). En 1997, des régions potentiellement métropolitaines ont été ajoutées à cette liste. De nombreuses études ont comparé les différentes fonctions métropolitaines des «Metropolregionen » et ont mis en avant l'importance que le label métropolitain avait prise dans le discours politique (Growe, Volgmann 2010 ; Blotevogel 2010 ; Schulz 2012 ; Hesse, Leick 2013). À partir de 2006, lors de la négociation d'un nouveau modèle d'aménagement fédéral, cette discussion a été étendue aux espaces frontaliers (en particulier Grande Région, Bodensee, Trinationale Metropolregion Oberrhein, Euregio Maas-Rhein; BBR, BMVBS 2006 : 8). À l'issue du 
projet MORO (Modellvorhaben der Raumordnung), la pertinence de définir une stratégie à part entière pour les régions métropolitaines transfrontalières a été confirmée (BVBS 2013). Depuis 2011, une instance spécifique, le Groupe d'Initiative « Régions métropolitaines transfrontalières » («Initiativkreis Metropolitane Grenzregionen », IMeG), est ainsi chargée d'assurer la valorisation et la prise en compte de ces espaces à l'échelle nationale et européenne.

- En Belgique comme plusieurs pôles métropolitains influençant directement le développement territorial sont situés en périphérie de son territoire (Aix-laChapelle, Bruxelles, Luxembourg, Lille et Maastricht), la fonction métropolitaine est interdépendante de la « coopération transrégionale » (SDER, 1999; SDER, 2013). La dimension transfrontalière apparaît de plus en plus comme une question incontournable dans la stratégie d'aménagement du territoire wallon puisque la dimension métropolitaine structure en partie la Wallonie, alors que celle-ci ne l'influence que partiellement. Dans le SDER, il s'agit donc de définir une approche à l'échelle régionale permettant de canaliser les retombées des métropoles voisines sur leur périphérie pour renforcer l'attractivité et le positionnement wallon en Europe. La dimension transfrontalière est mobilisée comme un levier pour influencer le développement des métropoles voisines (SDER, $2001: 3$ ).

- Le Luxembourg n'est pas en reste d'autant qu'un processus de métropolisation transfrontalier est à l'œuvre depuis les années 1990 et s'est amplifié au cours des années 2000. Il concerne essentiellement la ville de Luxembourg et le sud du pays. Le Programme Directeur d'Aménagement du Territoire adopté en 2003, document posant les orientations futures en matière d'aménagement du territoire, interprète la dimension métropolitaine comme un élément dynamique et structurant l'organisation et le rayonnement de l'espace. Prenant appui sur le SDEC, il dresse ainsi le constat qu' une dynamique très nette est présente dans les zones métropolitaines, alors que la Grande Région SaarLorLux ne présente cette tendance que «très localement (croissant Luxembourg-Metz-Sarrebruck) ( Programme directeur, 2003 : 154). Il constate par ailleurs que le « Grand-Duché n'est pas à même d'assumer à lui seul le développement de la [Grande Région SaarLorLux]. Une telle approche ne serait d'ailleurs pas souhaitable, les mesures mises en œuvre dans une optique nationale ne permettant pas d'exploiter des effets de synergies potentiels » (ibid.)

Le développement territorial du Luxembourg est donc interprété comme indissociable de celui de la Grande Région SaarLorLux. Sur base de ce constat, il propose de « définir au niveau de la [Grande Région SaarLorLux] un ensemble de réseau de villes aux fonctions différenciées et poursuivant des stratégies distinctes » (ibid.). Se dessine ainsi l'idée, comme dans la stratégie Metroborder, que les villes en Grande Région SaarLorLux doivent constituer 
une masse critique permettant de renforcer leur visibilité et leur poids européen. Sa réalisation implique, en l'occurrence, la dimension transfrontalière et, par voie de conséquence, la reconnaissance du caractère polycentrique de cet espace.

L'origine du positionnement métropolitain est donc à trouver dans l'existence de discours politiques concomitants et convergents aux niveaux européens et national mettant en avant la capacité d'attraction et d'innovation des métropoles. Si les quatre états développent des stratégies métropolitaines différentes (essentiellement juridique en France, instrumentale en Allemagne, orientés vers le transfrontalier au Luxembourg ou dans une moindre mesure en Belgique), il n'en demeure pas moins qu'un processus de métropolisation se structure en partie dans cet espace frontalier (Sohn, 2012), de même que dans de nombreux autres en Europe (Kujath, von Schlippenbach, 2002). Cette toile de fond coïncide avec, d'une part, la réalité bien connue des décideurs grand-régionaux d'interdépendances fonctionnelles transfrontalières grandissantes et toujours plus variées (Dörrenbächer, 2015 ; Belkacem, Pigeron-Piroth 2015) qui effectivement a été analysée comme un processus de métropolisation (Sohn 2012). D'autre part, ces discours métropolitains interviennent alors que la Grande Région SaarLorLux continue de rechercher une stratégie structurant l'ensemble de sa coopération (Wille 2009). Enfin, cette ambition métropolitaine se positionne opportunément par rapport aux priorités européennes posées notamment par l'Agenda territorial européen (Agenda territorial 2011). Ces différents éléments expliquent en partie l'ambition métropolitaine dont la Grande Région SaarLorLux s'est fait sienne. Ils illustrent aussi comment des discours aux échelles nationale et européenne influencent le positionnement d'une échelle transfrontalière en construction. Enfin, cette ambition métropolitaine pointe du doigt un débat récurrent ; celui de son espace effectif de coopération. En effet, si une partie de l'espace grand-régional, qualifié dans les débats institutionnels tantôt d' « espace noyau », tantôt de « cœur » (Evrard, à paraître), est caractérisée par un phénomène de métropolisation, la majeure partie de l'espace de coopération reste peu dense. Par conséquent, si l'ambition métropolitaine peut constituer une vision à laquelle la plupart des acteurs adhèrent (ESPON/Metroborder 2010), celle-ci est contrebalancée par la nécessaire reconnaissance de son caractère polycentrique.

\section{Réaffirmer le polycentrisme : la revendication d'un espace multipolaire}

Le concept de polycentrisme recouvre en Grande Région SaarLorLux plusieurs enjeux que l'étude ESPON, Metroborder a mis en évidence. En initiant la stratégie Metroborder en 2008, le Sommet des Exécutifs a commandé une étude auprès du programme européen ESPON (Observatoire en Réseau de l'Aménagement du Territoire Européen) afin d'identifier les potentiels d'une 
telle stratégie ainsi que des pistes de concrétisation. Pour résumer, cette étude ${ }^{2}$ (2009-2010) a essentiellement mis en avant trois éléments ayant un impact direct sur la façon d'opérationnaliser le concept de polycentrisme dans cet espace (représentés dans la carte 1).

\section{Carte 1 : Une région métropolitaine polycentrique transfrontalière au sein de la Grande Région SaarLorLux (ESPON, Metroborder 2010 : 13)}

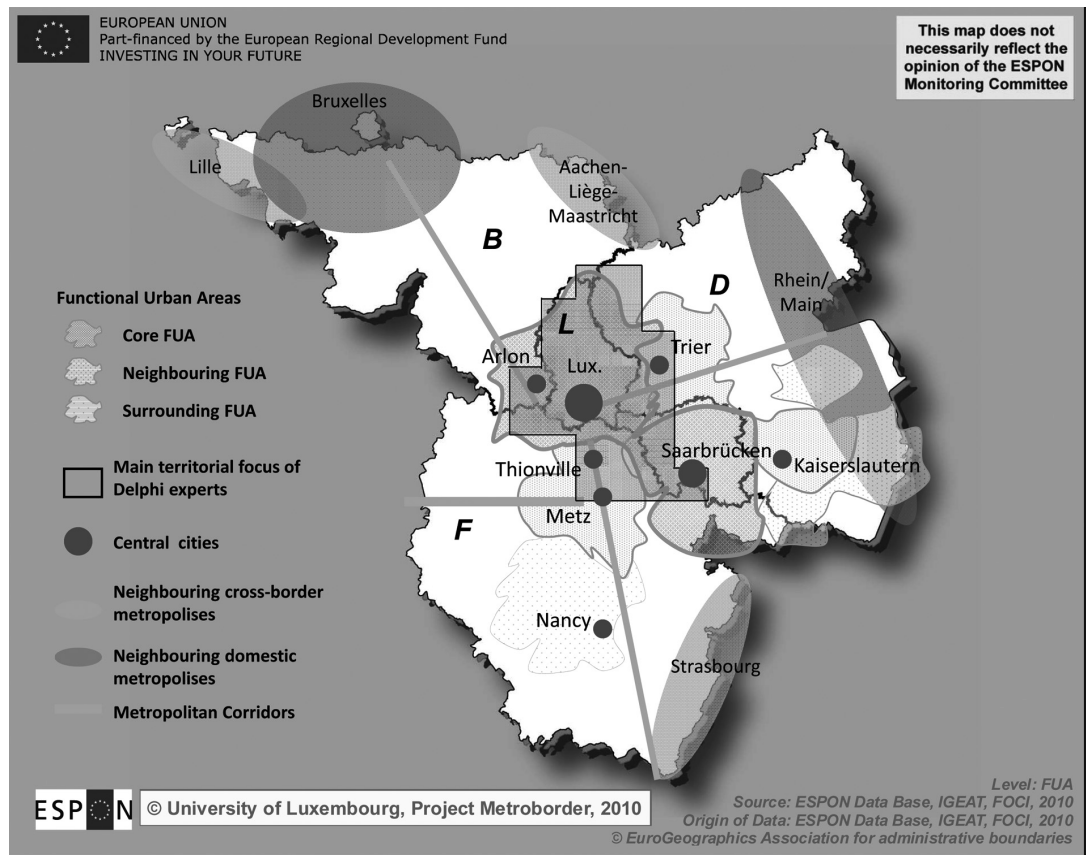

D'un point de vue fonctionnel, tout d'abord, la Grande Région SaarLorLux se caractérise par deux espaces urbains transfrontaliers, les agglomérations de Luxembourg et de Sarrebruck, presque adjacents d'un point de vue morphologique (ESPON, Metroborder 2010 : 23). Au sein de ces espaces, la plupart des interdépendances fonctionnelles transfrontalières se structurent, en particulier, en termes de travail frontalier. Cet espace métropolitain polycentrique comprend des villes telles qu'Arlon, Kaiserslautern, Luxembourg, Metz, Thionville, Trèves, Sarrebruck, Sarreguemines. Dans cet ensemble, l'agglomération de Luxembourg-ville apparaît prédominante économiquement, ce qui est renforcé par sa qualité de capitale nationale et européenne. En tant que capitales régionales, Sarrebruck et Metz, jouent également un rôle clé. Si ce cœur métropolitain compte plusieurs centres, chacun d'eux y joue un rôle spécifique.

2. Celle-ci a été réalisée par un consortium de chercheurs coordonnées par l'Université du Luxembourg et à laquelle les auteurs de cet article ont activement participé. 
En parallèle, l'espace de coopération tel que nous le connaissons actuellement (Schulz 2009), constitue l'espace de référence des acteurs institutionnels. Institutionnalisé depuis 1995 (avec une Wallonie observatrice à l'époque, membre à part entière depuis 2001), cet espace de coopération a régulièrement été confirmé depuis. D'un point de vue morphologique, cet espace de coopération se révèle être le plus vaste des espaces de coopération transfrontalière présentant un potentiel métropolitain (ESPON, Metroborder $2010: 53$ et 120). Pourtant, de fait, les acteurs associés à la coopération indiquent que la coopération transfrontalière en Grande Région SaarLorLux se concentre sur un « espace central » (ESPON, Metroborder $2010: 56)$; la carte d'éligibilité des projets INTERREG (2007-2013) illustre la reconnaissance institutionnelle implicite de cette réalité. Ce noyau (parfois nommé Kernraum par les acteurs institutionnels eux-mêmes) nuance l'espace métropolitain polycentrique identifié par l'analyse fonctionnelle puisqu'il diffère en fonction du domaine de coopération considéré. Il est particulièrement resserré pour ce qui est par exemple des transports de proximité, il est au contraire parfaitement inclusif pour ce qui est des lignes ferroviaires à grande vitesse (ESPON, Metroborder 2010 : 132). La coopération universitaire dans le cadre de l'Université de la Grande Région (UniGR) illustre un périmètre de coopération intermédiaire. Il en résulte que l'espace institutionnel de coopération, bien que resserré si l'on considère la coopération de proximité, est considéré par les acteurs politiques comme étant adéquat car reflétant l'engagement de l'ensemble des partenaires et permettant de manière pragmatique de coopérer dans de nombreux domaines (transports ferroviaires à grande vitesse, enseignement supérieur et recherche, aménagement du territoire, promotion économique). Un espace de coopération resserré ne pourrait permettre de traiter autant de thématiques de coopération. L'ambition polycentrique recouvre donc implicitement également une coopération transfrontalière sur plusieurs échelles, communale/locale ainsi que régionale.

Pour compléter ces différents éléments, force est de constater enfin, qu'en périphérie de la Grande Région SaarLorLux, des métropoles d'envergure nationale (Aix-la-Chapelle, Lille, Maastricht, Mannheim, Strasbourg), voire européenne (Bruxelles, Cologne, Francfort) exercent leur propre influence sur certains espaces grands-régionaux (ESPON, Metroborder 2010 : 13).

L'ambition métropolitaine met par conséquent directement en avant des enjeux et besoins de coopération grandissants (aménagement du territoire, marché du travail, transport), se manifestant essentiellement dans l'espace central de la coopération. Le terme " polycentrique » temporise ce constat de deux manières. $\mathrm{Au}$ sein de l'espace métropolitain central, ce terme vise à mettre en avant la complémentarité des différents centres urbains et donc des partenaires institutionnels. Le polycentrisme vise ensuite à garantir à l'ensemble des partenaires institutionnels que cette stratégie métropolitaine n'est pas exclusive d'autres domaines et thématiques de coopération s'inscrivant dans l'ensemble 
de l'espace grand-régional (tourisme, parcs naturels, enseignement supérieur et recherche, aménagement du territoire). Cette stratégie vient donc, avec précaution - comme en témoigne le terme « polycentrisme »-à coiffer d'un objectif de long terme (devenir métropolitain) des priorités de coopération thématiques (transport, aménagement du territoire, multilinguisme, mobilité professionnelle, recherche et enseignement supérieur) sur lesquelles, au demeurant, se concentraient déjà la plupart des projets de coopération.

En 2011, sous présidence sarroise, les Exécutifs ont «pris acte» des résultats de l'étude Metroborder, indiquant que la mise en œuvre de cette stratégie « pourra renforcer et développer [la] compétitivité [de la Grande Région SaarLorLux] en tant que région métropolitaine polycentrique transfrontalière au sein de l'ensemble des régions métropolitaines européennes » (Sommet 2011: 9). Depuis 2009, Metroborder apparaît donc comme un projet politique clé pour les partenaires institutionnels, il participe au processus de construction de l'entité transfrontalière qu'est la Grande Région SaarLorLux. Au-delà de ces ambitions politiques, nous proposons d'en évaluer la réalité opérationnelle à travers deux réalisations récentes.

\section{- Le lancement à petits pas de la stratégie Metroborder}

Dans leur déclaration commune de 2011, les Exécutifs fixent deux grands objectifs comme moyen de concrétiser la stratégie Metroborder. Dans la continuité de ce qui avait été décidé entre 2008, ils visent l' "élaboration d'une stratégie de développement territorial » et le « développement d'une gouvernance multi-niveaux » (Sommet 2011 : 19). Deux jalons ont principalement été posés dans ce sens ; la création d'un groupement européen de coopération territoriale (GECT) et d'un système d'information géographique (SIG).

\section{Une timide institutionnalisation de la coopération : le GECT Secrétariat du Sommet}

Jusqu'à la mise en place du GECT « Secrétariat du Sommet de la Grande Région » (GECT/Sommet), nous pouvons estimer que malgré les nombreuses instances de coopération qu'elle compte (Clément 2015), la coopération en Grande Région est relativement peu institutionnalisée. Cette affirmation est essentiellement étayée par un constat; les différentes instances de coopération fonctionnent sur base de la collégialité des membres (décisions à l'unanimité), la présidence de l'institution étant assurée successivement par ses membres (sur le modèle du Conseil européen). Aucune institution commune n'est en charge de coordonner et d'assurer le suivi des décisions communes. Dans ce contexte, le suivi des activités entre chaque présidence et la coordination 
entre les différentes instances de coopération représentent des défis constants, d'autant que les partenaires associés sont nombreux.

Le GECT Sommet constitue dans ce contexte une avancée significative. Premier outil juridique mis en place par l'Union européenne, il vise à faciliter et promouvoir la coopération territoriale en Europe (Parlement européen et Conseil 2006). Disposant de la personnalité juridique et pouvant ainsi conduire ses propres projets, le GECT peut se voir conférer des compétences qu'il exerce au nom et pour le compte de ses membres, situés dans différents États membres (ibid. $)^{3}$. Cette structure n'a bien évidemment aucune incidence sur les asymétries institutionnelles et juridiques entre les partenaires (vide institutionnel) identifiées plus haut. Par contre, en la mettant sur pied, les partenaires formalisent la coopération antérieure. Ils vont au-delà des « routines » et « modes de régulation » établis au fil du temps, visant, alors qu'aucun cadre ne préexiste, à coordonner leur coopération, à lui allouer des ressources et à prévenir ou résoudre des conflits (March, Olsen 1989 : 483). En créant cette structure, ils s'engagent les uns envers les autres juridiquement et financièrement sans limite temporelle. Cette structure présente donc le potentiel de combler partiellement un vide institutionnel au niveau transfrontalier et interrégional.

Tab. 1 : Les principales caractéristiques du GECT Secrétariat du Sommet

\begin{tabular}{|l|l|}
\hline Siège & Maison de la Grande Région, Esch-S.-Alzette, LU \\
\hline Droit & Luxembourgeois (art. 5) \\
\hline Durée & Établi pour une durée illimitée (art. 6) \\
\hline $\begin{array}{l}\text { Organe } \\
\text { décision- } \\
\text { naire }\end{array}$ & $\begin{array}{l}\text { Assemblée générale réunissant l'ensemble des partenaires institutionnels } \\
\text { (art. 13, statuts), présidée par son directeur }\end{array}$ \\
\hline Directeur & $\begin{array}{l}\text { Représentant en charge de la Présidence tournante du Sommet (art. 14, } \\
\text { statuts) }\end{array}$ \\
\hline $\begin{array}{l}\text { Mode de } \\
\text { prise de } \\
\text { décision }\end{array}$ & $\begin{array}{l}\text { Chacune des « cinq composantes territoriales » institutionnnalisées (Lor- } \\
\text { raine, Luxembourg, Rhénanie-Palatinat, Sarre, Wallonie, art.1 statuts) } \\
\text { dispose de 4 voix, les décisions sont prises à la majorité de 3 compo- } \\
\text { santes territoriales (réunissant 11 voix ou plus, art. 13.1, statuts) }\end{array}$ \\
\hline $\begin{array}{l}\text { Gestion } \\
\text { opération- } \\
\text { nelle }\end{array}$ & $\begin{array}{l}\text { Le gérant " assure les fonctions de gestion administrative et logistique } \\
\text { quotidienne du secrétariat » (art. 14, statuts). }\end{array}$ \\
\hline
\end{tabular}

a. Font exception et sont soumises à l'unanimité les décisions concernant l'adhésion de nouveaux membres (articles 9.2), la perte de la qualité de membre (art.10), l'exclusion d'un membre (art.11), le budget (art.18), la modification des statuts (art.19) et la dissolution du GECT (art.20).

3. La Grande Région SaarLorLux a déjà eu recourt à cet instrument à deux reprises : le GECT Alzette Belval (Clément 2015) et le GECT Autorité de gestion du programme INTERREG IVA Grande Région a assuré la mise en œuvre opérationnelle du programme. 
Légalement constitué en 2013, le GECT Sommet a recruté son personnel et lancé ses activités courant 2014. Nous ne disposons donc pas du recul suffisant pour pouvoir analyser son impact sur la coopération. Toutefois, la convention l'instituant ainsi que ses statuts sont d'importants éléments permettant de prendre la mesure de ses futures marges de manœuvre. Il apparaît assez clairement à la lecture de ces documents, que le GECT a été établi afin de faciliter et d'améliorer le fonctionnement opérationnel du GECT (suivi et mise en œuvre des décisions, coordination des groupes de travail). Incidemment, il formalise également le fonctionnement du Sommet autour d'une présidence ayant le pouvoir de définir l'agenda et d'orienter la coopération fonctionnant par rotation tous les deux ans (tableau 1).

Ces caractéristiques invitent à formuler plusieurs remarques :

- Directeur : dans la lignée de l'usage qui préexistait, le directeur du GECT reste un représentant politique issu de l'une des entités partenaires. Généralement élu, il dispose d'une légitimité, d'un poids politique et d'une certaine visibilité extérieure. Cela peut contribuer à mieux faire connaitre les activités du Sommet à l'extérieur, bien qu'une présidence tournante atténue son impact, en particulier sur le long terme.

- Mode de prise de décision : en renonçant à l'unanimité, certaines situations de blocage dans la gestion quotidienne du GECT devraient être évitées. Jusqu'à présent, les partenaires avaient exclu de travailler par «versant » ou composante territoriale (sauf pour les questions budgétaires et pour la présidence du sommet). Les raisons à cela sont nombreuses, la plus importante étant certainement le principe du respect de l'organisation institutionnelle de chaque région (Evrard à paraitre). L'institutionnalisation de ce système de vote favorise mécaniquement les composantes représentant un seul partenaire (Luxembourg, Sarre, Rhénanie-Palatinat qui à eux trois pourraient par exemple faire adopter des décisions). Elle incite en conséquence les membres d'une même composante à coordonner leur décision en amont des assemblées générales afin de renforcer leur positionnement. Cela vaut essentiellement pour la Lorraine impliquant quatre entités. Sur le long terme, cela pourrait favoriser l'émergence d'une vision propre à chaque composante de l'espace frontalier. En somme l'asymétrie institutionnelle entre partenaires serait déplacée du niveau transfrontalier pour être traitée au niveau régional.

- Le/a gérant(e) : ses fonctions sont comprises de manière restrictive, elles sont essentiellement techniques et logistiques, les rôles de mise en œuvre des décisions communes ou encore de proposition sont clairement exclus.

Les statuts prévoient cependant une évaluation de ses activités après quatre ans, ce qui peut donner lieu soit à « une extension soit à une réduction » de ses missions (art. 2, statuts). Cette disposition révèle une certaine prudence à l'égard de l'institutionnalisation de la coopération. 
Dans l'immédiat, cette nouvelle structure a un impact limité sur la stratégie Metroborder, dans la mesure où elle ne participe pas directement à sa concrétisation. Toutefois, cette stratégie s'inscrit à la charnière de nombreuses thématiques de la coopération (aménagement du territoire, transport, enseignement et recherche), son rôle de suivi et de coordination des travaux des groupes de travail pourrait se révéler crucial sur le long terme. Jusqu'à présent, l'absence de structure permanente commune pouvait conduire à une certaine isolation des groupes thématique de travail (Evrard à paraître).

Le caractère restrictif des missions confiées au GECT montre que les partenaires institutionnels entendent conserver une large marge de manœuvre sur cet instrument qui incarne la construction politique de la Grande Région SaarLorLux. Le GECT pourrait se révéler être un rouage essentiel de la coopération transfrontalière dans la mesure où il institutionnalise au niveau politique le processus de prise de décision des partenaires. Au niveau administratif, il assure le rôle de courroie de transmission entre les partenaires et entre les groupes de travail. Les prochaines années seront cruciales puisqu'elles révéleront l'interprétation que les partenaires et le personnel nouvellement recruté réserveront à ces textes.

\section{Le SIG-GR : appréhender les développements territoriaux pour mieux asseoir les décisions communes}

En parallèle de la stratégie Metroborder initiée en 2009, le Sommet a lancé la mise en place d'un système d'information géographique de la Grande Région (SIG-GR) (Sommet 2009). Financé d'abord sous la forme d'un projet INTERREG (2010-2013), il a ensuite été prolongé en tant qu' « outil permanent et commun de travail » (Présidence luxembourgeoise 2009: 16), financé par l'ensemble des partenaires. La pérennisation de ce projet souligne le caractère crucial, voire stratégique, de la coopération dans le domaine de l'aménagement du territoire. Si une «démarche commune et cohérente » n'existe pas encore dans ce domaine, le SIG-GR constitue une première étape qui semble prometteuse à deux égards.

Faisant face à des systèmes statistiques régionaux hétérogènes, le SIG GR vise à mettre en place des indicateurs, définitions et outils de visualisation transfrontaliers. « À caractère analytique, [il] doit permettre de rassembler et d'harmoniser les données existantes, mais surtout de développer des indicateurs territoriaux au niveau grand-régional » (Présidence luxembourgeois 2009: 19). Le Sommet emploie le terme de « langage commun » visant à mieux appréhender les développements territoriaux transfrontaliers (ibid. : 17). Ce projet dénote la volonté de s'approprier en commun l'espace transfrontalier par le biais d'outils de connaissance. L'enjeu est, en amont d'un processus décisionnel, de disposer 
d'une base d'information commune objective et donc de dépasser une connaissance individuelle et personnelle de l'espace transfrontalier. Il s'agit d'une appropriation cognitive de l'espace transfrontalier (Ripoll, Veschambres, 2005 ; Evrard à paraître). Publiquement accessible en ligne, l'objectif est également de viser à renforcer la visibilité de la Grande Région SaarLorLux vers l'extérieur (Présidence luxembourgeoise 2009: 17). En ce sens, ce projet a pour but de visualiser et, par là même, de donner substance à une réalité transfrontalière vécue ou restée floue. Cet outil inscrit une réalité fonctionnelle, tangible, objective dans un cadre construit par des décisions politiques. Il constitue un pendant objectif aux perceptions individuelles, nécessairement subjectives, tant des acteurs politiques régionaux que de tout autre acteur institutionnel et des citoyens. Ainsi, il participe à la construction cognitive d'un espace transfrontalier commun.

C'est ensuite, précisément parce qu'elle a été menée au nom et pour le compte de l'ensemble des acteurs - donc objectivement - que cette appropriation cognitive peut servir à « appuyer la poursuite de la démarche d'informationconsultation-concertation en matière d'aménagement du territoire » (Présidence luxembourgeoise 2009: 14). Le SIG optimise en effet la « compréhension des dynamiques territoriales observées dans la Grande Région. Leurs représentations cartographiques orientent les politiques de développement et d'aménagement du territoire et guident les acteurs dans leur prise de décision en permettant, à terme, une meilleure maîtrise de la diversité régionale » (ibid.).

L'objectif ultime est l' « harmonisation de la planification territoriale au sein de la Grande Région qui se ferait sur la base d'une meilleure concertation lors de l'élaboration des différents documents de planification à impact transfrontalier et/ou grand-régional réalisés par les entités membres » (Présidence luxembourgeoise 2009: 17). In fine, cette appropriation cognitive de l'espace opérée par le SIG-GR, permettrait aux partenaires institutionnels de prendre en commun des décisions dont la mise en œuvre ensuite est régionale (appropriation matérielle, Ripoll, Veschambres, 2005). L'échelle transfrontalière est ainsi progressivement institutionnalisée comme lieu d'information, d'échange et de prises de décision en commun ; elle supplée l'échelle régionale sans la remplacer. Les décisions restent mises en œuvre au niveau régional, sur la base d'un engagement politique non contraignant - étant donné le caractère intrinsèquement volontaire de la coopération transfrontalière.

Si ce projet constitue incontestablement une avancée pour la coopération en matière d'aménagement du territoire, notons que le Rhin supérieur dispose d'un SIG équivalent depuis 2000 . 


\section{- Conclusion}

Après de longues années de coopération, la Grande Région SaarLorLux entend ancrer sa stratégie de développement transfrontalière autour d'une ambition métropolitaine. Aussi paradoxale que cette ambition puisse sembler dans ce vaste espace transfrontalier globalement peu dense, ce positionnement relève avant tout d'un positionnement stratégique vis-à-vis des échelles nationale et européenne tout en visant à structurer une stratégie commune d'aménagement du territoire. Comme dans d'autres espaces frontaliers, la coopération dans le domaine de l'aménagement du territoire constitue à la fois une nécessité - étant donné les interdépendances fonctionnelles toujours plus variées et importantes - et un défi permanent compte tenu des asymétries institutionnelles, différences linguistiques, législatives et administratives. Ces difficultés sont accrues dans le contexte grand-régional qui compte le plus grand nombre de travailleurs frontaliers en Europe, engendrant des interdépendances fonctionnelles complexes. Ces éléments expliquent en partie le piétinement de la coopération en matière d'aménagement du territoire et, en conséquence, le lancement de la stratégie Metroborder. Cette dernière révèle la tentative des acteurs politiques de construire, sur le long terme, une vision politique commune de l'espace de coopération, l'aménagement du territoire constituant le domaine embrassant la coopération dans son ensemble. Depuis son lancement en 2008, force est de constater que la mise sur pied du GECT et le projet SIG constituent des étapes importantes mais insuffisantes dans l'opérationnalisation de cette dernière. Essentiellement volontaire, la coopération transfrontalière est profondément tributaire de l'engagement politique. Fluctuant au niveau national, il l'est d'autant plus dans le contexte transfrontalier. Dans ce contexte, l'institutionnalisation de la coopération par le biais d'organes indépendants, disposant d'un mandat clairement défini peut être gage de constance et de régularité. Après avoir fondé pendant de longues années sa coopération sur un travail interrégional, il semble que, très timidement, la Grande Région SaarLorLux s'oriente vers la voie de l'institutionnalisation. La réforme institutionnelle en cours du côté français et la possible constitution d'une région « Alsace - Lorraine - Champagne - Ardennes » pourrait à tout le moins ralentir ce processus, en tout cas, introduire une complexité institutionnelle supplémentaire à l'architecture grand-régionale. Si cette région des « quatre frontières » devait jouer indéniablement un rôle d'interface clé avec ces voisins européens, elle signifierait aussi pour la Grande Région SaarLorLux un transfert de pouvoir de Metz vers Strasbourg. 


\section{$\boldsymbol{V}$ Bibliographie}

Affolderbach J. (2013), « Negotiating Border Regions. Retail Development in Luxembourg and the Greater Region », dans : Gilles P., Koff H., Maganda C. et Schulz Ch. (éd.), Theorizing Borders Through Analyses of Power Relationships. Brussels : PIE Peter Lang, p. 125-148.

Agenda territorial 2020 (2011), Territorial agenda of the European Union 2020 - Towards an Inclusive, Smart and Sustainable Europe of Diverse Regions, agreed at the Informal Ministerial Meeting of Ministers responsible for Spatial Planning and Territorial development, http://www.eu2011.hu/files/bveu/documents/TA2020, consulté le 26.02.2013.

BBR/BMVBS (éd.) (2006), Perspektiven der Raumentwicklung in Deutschland, Bonn/Berlin.

Belkacem R. et Pigeron-Piroth I. (2015), « Un marché de l'emploi intégré ? L'emploi frontalier et ses dimensions socioéconomiques » dans : WILLE Ch. (éd.), Lebesnwirklichkeiten und politische Konstruktionen in Grenzregionen. Das Beispiel der Großregion SaarLorLux : Wirtschaft - Politik Alltag - Kultur, Transcript.

Blatter J. (2004), « From "Spaces of Place" to "Spaces of Flows"? Territorial and Functional Governance in Cross-border Regions in Europe and North America ». International Journal of Urban and Regional Research 28 (3), p. 530-548.

BMVBS (2013), Initiativkreis deutscher Regionen in grenzüberschreitenden Verflechtungsräumen, http://www.metropolitane-grenzregionen.eu/public/filecache/MORO_IMeG_Final_ Report_2013.pdf, consulté le 27.11.2014.

Blotevogel H-H. (2010), « Raumordnung und Metropolregionen ». Geographischer Rundschau 62 (11), S. 4-13.

BmBau - Bundesministerium für Raumordnung, Bauwesen und Städtebau (éd.) (1995), Raumordnungspolitischer Handlungsrahmen : Beschluss der Ministerkonferenz für Raumordnung am 08.03.1995, Bonn.

Chilla T., Evrard E. et Schulz Ch. (2012), « On the Territoriality of Cross-Border Cooperation : “Institutional Mapping" in a Multi-Level Context ». European Planning Studies 20 (6), p. 961980.

Chilla T. et Schulz Ch. (2014), «Spatial Development in Luxembourg: Mimetic Evolution or Emergence of a New Planning Culture ? » European Planning Studies.

Clément F. (2015), « La représentation politique dans la Grande Région SaarLorLux : institutions, compétences et coopérations », dans : WILLE Ch. (éd.), Lebesnwirklichkeiten und politische Konstruktionen in Grenzregionen. Das Beispiel der Großregion SaarLorLux : Wirtschaft - Politik Alltag - Kultur, Transcript.

Comité de Coordination du Développement Territorial (CCDT) (2012), Schéma de développement territorial de la Grande Région. Volet 1: la dimension métropolitaine de la Grande Région, http://www.dat.public.lu/actualites/2013/01/1701_Reunion_sectorielle_ Grande_Region/2801_Schema_de_developpement_territorial_GR.pdf, consulté le 27.11.2014.

Commission européenne (2014), Aide d'Etat: La Commission adopte une série de décisions concernant des aides publiques en faveur d'aéroports et de compagnies aériennes en Belgique, en Allemagne, en Italie et en Suède, http://europa.eu/rapid/press-release_IP-14-1065_fr.htm, consulté le 01.12.14.

Comte H. et Levrat N. (2006), Aux coutures de l'Europe - Défis et enjeux juridiques de la coopération transfrontalière, Paris : L'Harmattan. 
CGCT (Code général des collectivités territoriales), version consolidée au 20 janvier 2013, www.legifrance.fr, consulté le 27.11.2014.

Dörrenbächer P. (2015), « Ein grenzüberschreitender Wirtschaftsraum ? Gemeinsame und individuelle Entwicklungen seit der Krise des Montansektors », dans : WILLE Ch. (éd.), Lebenswirklichkeiten und politische Konstruktionen in Grenzregionen. Das Beispiel der Großregion SaarLorLux : Wirtschaft - Politik - Alltag - Kultur, Transcript.

Dühr S., Colomb C. et Nadin V. (2010), European Spatial Planning and Territorial Cooperation, London/New York : Routledge.

ESPON/Metroborder (2010), Cross-border polycentric metropolitan regions - final report, http://www.espon.eu/export/sites/default/Documents/Projects/TargetedAnalyses/ METROBORDER/METROBORDER_-_Final_Report_-_29_DEC_2010.pdf, consulté le 26.02.2013.

Evrard E. (à paraître), Suprarégionalisation transfrontalière ? Grande Région SaarLorLux. Rennes : Presses universitaires de Rennes.

Faludi A. (éd.) (2008), European spatial research and policy. Cambridge MA, USA : Lincoln Institute of Land Policy.

Growe A. et Volgmann K. (2010), « Die deutschen Metropolräume im globalen Standortwettbewerb », Raum Planung (153), p. 259-299.

Hajer M. (2003), « Policy without Polity ? Policy analysis and the institutional void ». Policy Sciences 36 (2), p. 175-195.

Hinfray N. (2010), Les espaces transfrontaliers, nouveaux territoires de projet, nouveaux pôles de développement au sein d'une Europe polycentrique. Thèse de doctorat sous la direction de Jean-Paul Carrière, Université de Tours

Hooghe L. et Marks G. (2003), « Unravelling the Central State, but How ? Types of Multi-level Governance ». American Political Science Review 97 (2), p. 233-243.

Hoyler M., Freytag T. et Mager Ch. (2006), «Advantageous fragmentation ? Reimagining metropolitan governance and spatial planning in Rhine-Main ». Built Environment 32 (2), p. 124-136.

Juncker J-C. (2006), Déclaration du gouvernement sur la situation économique, sociale et financière du pays 2006, http://www.gouvernement.lu/gouvernement/etat-nation/2006/ index.html.

Knippschild R. (2011), « Cross-Border Spatial Planning : Understanding, Designing and Managing Cooperation Processes in the German-Polish-Czech Borderland ». European Planning Studies 19 (4), p. 629-645.

Kujath H-J. et Von Schlippenbach U. (2002), « Europäische Verflechtungen deutscher Metropolregionen als Herausforderung für Politik und Wirtschaft - Ergebnisse einer Fachkonferenz ». Informationen für Raumentwicklung 6/7, p.381-392.

Leber N. et Kunzmann K-R. (2006), « Entwicklungsperspektiven ländlicher Räume in Zeiten des Metropolfiebers ». disP 3 (166), p. 58-70.

March J-G. et Olsen J-P. (1989), Rediscovering institutions. The organizational basis of politics, New York : Free Press/Macmillan.

Ministère des transports du Grand-duché de Luxembourg, Conseil régional de Lorraine, CFL, SNCF (2008), Diagnostic socioéconomique et analyse de la mobilité transfrontalière LorraineLuxembourg. Présentation des conclusions, Luxembourg. 
Niedermeyer M. et Moll P. (2007), « SaarLorLux - vom Montandreieck zur "Großregion". Chancen und Möglichkeiten einer grenzüberschreitenden Regionalpolitik in Europa », dans Dörrenbächer H-P., Kühne 0. et Wagner J-M. (éd.), 50 Jahre Saarland im Wandel. Saarbrücken : IfLiS, p. 297321.

Othengrafen F. et Reimer M. (2013), « The embeddedness of planning in cultural contexts : theoretical foundations for the analysis of dynamic planning cultures ». Environment and Planning A 45 (6), p. 1269-1284.

Parlement européen et Conseil (2006), Regulation $n^{\circ} 1082 / 2006$ on a European Grouping of territorial cooperation (EGTC), Official journal of the European Union L 210, p. 19-24.

Perkmann M. (2003), « Cross-Border Regions in Europe : Significance and Drivers of Regional Cross-Border Co-0peration », European Urban and Regional Studies 10 (2), p. 153-171.

Perkmann M. (2007a), «Policy entrepreneurship and multilevel governance : a comparative study of European cross-border regions ». Environment and Planning $C$ : Government and Policy 25 (6), p. 861-879.

Perkmann M. (2007b), Construction of New Territorial Scales : A Framework and Case Study of the EUREGIO Cross-border Region. Regional Studies 41 (2), p. 253-266.

Présidence luxembourgeoise du 11ème Sommet de la Grande Région (2009), Le développement et la planification territoriale au niveau de la Grande Région. Rapport d'activités du volet « ESPACE » - Programme de travail de la présidence luxembourgeoise du 11ème Sommet, http://www.granderegion.net/fr/documents-officiels/declarations-communes-finales/ 11_SOMMET_RAPPORT_ACTIVITES_VOLET_I_ESPACE.pdf, consulté le 27.11.2011.

Programme Directeur d'Amenagement du Territoire (2003), Ministère de l'Intérieur, Direction de l'aménagement du territoire et de l'urbanisme, adopté par le gouvernement luxembourgeois le 27.03.2003, http://www.dat.public.lu/publications/documents/programme_ directeur/programme_directeur_2003_fr_partie_a_hr.pdf, consulté le 26.02.2013.

Renard J-P. (2007), «Frontières et aménagement : le point de vue du géographe ». Mosella 32 (1-4), p. 5-16.

Ripoll F. et Veschambre V. (2005), « L'appropriation de l'espace comme problématique », Norois, 195 (2), p. 2-10

Ritter E-H. (2010), Europäische Raumentwicklungspolitik : Inhalte, Akteure, Verfahren, Organisation. Rohn

Schulz Ch. (1999), « City-networking and bottom-up development by transfrontier co-operation », dans : Knippenberg H. et Markusse J. (éd.), Nationalising and Denationalising European Border Regions (1800-2000). Views from Geography and History. Dordrecht: GeoJournal Library 53, p. 223-240.

Schulz Ch. (2009a), Luxembourg dans la «Grande Région » dans : Bousch P., Chilla T., Klein 0., Gerber P., Schulz Ch., Sohn Ch. et Wiktorin D. (éd.), L'atlas du Luxembourg - Luxemburg Atlas. Köln : Emons, p.12-13.

Schulz Ch. (2012), "La politique des “Metropolregionen" en Allemagne », Bulletin de l'Association de Géographes français 89 (4), p. 512-522.

Schulz Ch. (2013), « Power Topographies in Cross-Border Spatial Development Policies » dans Gilles P., Koff H., Maganda C. et Schulz Ch. (éd.), Theorizing Borders Through Analyses of Power Relationships. Brussels : PIE Peter Lang, p. 111-123. 
SDEC (1999), Schéma de Développement de l'Espace Communautaire - Vers un développement spatial équilibré et durable du territoire de l'Union européenne, Approuvé au Conseil informel des Ministres responsables de l'aménagement du territoire de Potsdam, Luxembourg.

SDER (1999), Schéma de Développement de l'Espace Régional, www.sder.wallonie.be, consulté le 27.11.2014.

SDER (2001), Fiche thématique 2, http://sder.wallonie.be/Dwnld/Fiches/Fiche\%2002.pdf, consulté le 26.02.2013

SDER (2013), Schéma de développement de l'espace régional, projet adopté par le Gouvernement Wallon le 7.11.2013, http://spw.wallonie.be/dg04/sder/dwnld/5806-sderprojetbr1.pdf, consulté le 27.11.2014.

Sohn Ch. (éd.) (2012), Luxembourg - an emerging cross-border metropolitan Region. Brussels : Peter Lang.

Sommet des Exécutifs de la Grande Région (2008), $10^{\text {e }}$ Sommet de la Grande Région - Déclaration commune, Namur.

Sommet des Exécutifs de la Grande Région (2009), $11^{\text {e }}$ Sommet de la Grande Région, Déclaration commune, http://www.granderegion.net/fr/news/2009/07/e_20090717/index.html, consulté le 03.12.2014.

Sommet des Exécutifs de la Grande Région (2011), $12^{\mathrm{e}}$ Sommet de la Grande Région, Déclaration commune, http://www.mwkel.rlp.de/File/12-Gipfel-Grossregion-2011-GemeinsameErklaerung-pdf/, consulté le 03.12.2014.

Spannowsky W. (éd.) (2005), Die Ansiedlung von großflächigen Einzelhandelsbetrieben. Vergleich der räumlichen Planungssysteme in der Großregion Saarland, Lothringen, Großherzogtum Luxemburg, Region Wallonien und Rheinland-Pfalz (= Schriftenreihe zum Raumplanungs-, Bau- und Umweltrecht, Band 6), Kaiserslautern : TU Kaiserslautern, p. 299.

Spannowsky W. (2007), Vergleich der räumlichen Planungssysteme in der Großregion Saarland, Lothringen, Großherzogtum Luxemburg, Region Wallonien und Rheinland-Pfalz. Abschlussbericht, Kaiserslautern : TU Kaiserslautern.

STATEC (éd.) (2009), Le Luxembourg en chiffres, Luxembourg.

Statuts du Groupement Européen de coopération territoriale (GECT) « Secrétariat du Sommet de la Grande Région ». Mémorial $n^{\circ} 155$ (28 août 2013), p. 3000.

Sykes 0. et Shaw D. (2008), «Investigating Territorial Positioning by Sub-state Territories ». Europe, Regional \& Federal Studies 18 (1), p. 55-76.

Vidal M-J. et Niedermeyer M. (2011), « Le développement territorial et sa dimension transfrontalière. Expériences du Luxembourg et de la Grande Région » dans : Chilla T. et Schulz Ch. (éd.), Raumordnung in Luxemburg - Aménagement du territoire au Luxembourg. Luxembourg : Editions Binsfeld, p. 296-316.

Waterhout B. (2008), The institutionalisation of European spatial planning. Delft University Press. Wille Ch. (2009), « Eine namenlose Region ». Forum (288), p. 30-31.

Wille Ch. (2008), « Zum Modell des transnationalen sozialen Raums im Kontext von Grenzregionen. Theoretisch-konzeptionelle Überlegungen am Beispiel des Grenzgängerwesens ». Europa Regional 16 (2), p. 74-84.

Wille Ch. (2015), «Grenzüberschreitende Alltagspraktiken in der Großregion SaarLorLux : eine Bestandaufnahme », dans : WILLE Ch (ed.), Lebesnwirklichkeiten und politische Konstruktionen in Grenzregionen. Das Beispiel der Großregion SaarLorLux : Wirtschaft - Politik - Alltag - Kultur, Transcript. 